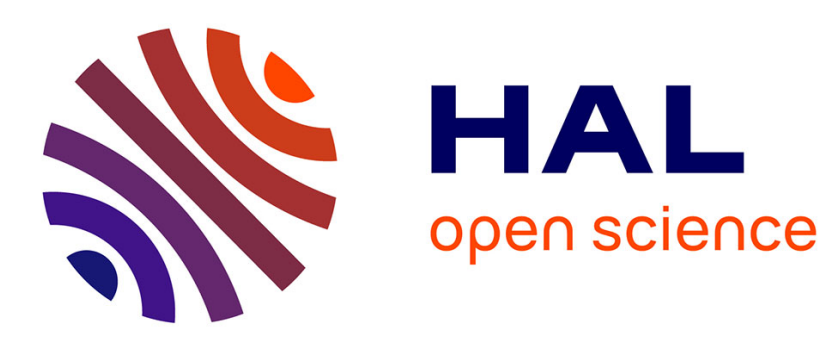

\title{
Existence and regularity results for fully nonlinear equations with singularities
}

\author{
Patricio Felmer, Alexander Quaas, Boyan Sirakov
}

\section{To cite this version:}

Patricio Felmer, Alexander Quaas, Boyan Sirakov. Existence and regularity results for fully nonlinear equations with singularities. Mathematische Annalen, 2012, 354 (1), pp.377-400. hal-00462082v2

\section{HAL Id: hal-00462082 \\ https://hal.science/hal-00462082v2}

Submitted on 7 Jul 2010

HAL is a multi-disciplinary open access archive for the deposit and dissemination of scientific research documents, whether they are published or not. The documents may come from teaching and research institutions in France or abroad, or from public or private research centers.
L'archive ouverte pluridisciplinaire HAL, est destinée au dépôt et à la diffusion de documents scientifiques de niveau recherche, publiés ou non, émanant des établissements d'enseignement et de recherche français ou étrangers, des laboratoires publics ou privés. 


\title{
Existence and regularity results for fully nonlinear equations with singularities
}

\author{
Patricio Felmer, Alexander Quaas and Boyan Sirakov
}

\begin{abstract}
In this paper we consider the Dirichlet boundary value problem for a singular elliptic PDE lke $F[u]=p(x) u^{-\mu}$, where $p, \mu \geq 0$, in a bounded domain in $\mathbb{R}^{n}$. The nondivergence form operator $F$ is assumed to be of Hamilton-Jacobi-Bellman or Isaacs type. We establish existence and regularity results for such equations.
\end{abstract}

\section{Introduction}

In this paper we study a class of boundary value problems of the form

$$
F\left(D^{2} u, D u, u, x\right)+f(x, u)=0 \quad \text { in } \quad \Omega, \quad u=0 \quad \text { on } \quad \partial \Omega,
$$

where $F$ is a positively homogeneous fully nonlinear elliptic operator, $\Omega$ is a bounded $C^{2}$-domain and $f$ is singular at $u=0$. Our main results concern existence, uniqueness and regularity of solutions of (1.1).

The semi-linear version of this problem, that is, (1.1) with $F$ replaced by a linear operator

$$
L=a_{i j}(x) \partial_{x_{i} x_{j}}^{2}+b_{i}(x) \partial_{x_{i}}+c(x),
$$

has been attracting continuous attention since the 1970's and the literature nowadays is very large. A cornerstone in the study of singular problems of this type is the classical work by Crandall, Rabinowitz and Tartar [10], whose results, applied to the model equation

$$
L u+p(x) u^{-\mu}=0 \quad \text { in } \quad \Omega, \quad u=0 \quad \text { on } \quad \partial \Omega,
$$

$\mu>0, p(x)>0$ in $\bar{\Omega}$, state that (1.3) has a unique positive solution which is Hölder continuous, that is $u \in C^{\gamma}(\bar{\Omega})$, and it satisfies

$$
C_{1} d^{\gamma}(x) \leq u(x) \leq C_{2} d^{\gamma}(x)
$$

where $d$ is the distance to the boundary of $\Omega, \gamma=2 /(\mu+1)$ and $0<C_{1} \leq C_{2}$. In addition, they showed that if $\mu<1$ then the solution is Lipschitz in $\bar{\Omega}$. Later, Lazer and McKenna [25] developed a simplified approach to this problem, in particular when $L$ is in divergence form.

Concerning the regularity of solutions of (1.3), exact results were obtained by del Pino [12] and Gui-Lin [18], for $L=\Delta$ and functions $p$ which behave 
like powers of the distance to the boundary. In particular, they showed that solutions of (1.3) are actually in $C^{2}(\Omega) \cap C^{1, \beta}(\bar{\Omega})$, for some $\beta>0$, provided $\mu<1$ (see also an earlier result by Gomes [17]). Notice that this is the best regularity one can expect, as the equation itself shows.

Then, in the last fifteen years numerous and various extensions of these results were obtained, mostly to more general nonlinearities $f(x, u)$ and to quasilinear equations. In addition to the papers we already quoted we refer to [6], [7], [11], [13], [20], [21], [31], [34], [37], [38] and the references therein. An excellent starting point to these studies is the survey [19], where very extensive literature is available.

Despite the large literature on semi- and quasi-linear equations with singular nonlinearities, we do not know of any works where such a study is done in the setting of fully nonlinear equations. This is the main goal of this paper. We are going to show that the recently developed theory of HamiltonJacobi-Bellman and Isaacs equations [8], [22], [5], [9], [29] and [1] permits us to prove the same existence results as in the semi-linear setting, when weak (viscosity) solutions to (1.1) are considered. The regularity result requires a new approach, since none of the methods used in the previous papers on singular problems applies in the fully nonlinear setting. In particular, no linear theory for adjoint operators nor Green functions are available for fully nonlinear operators.

Throughout the paper all differential (in)equalities will be understood to hold in the $L^{N}$-viscosity sense - see [5] for a detailed description of this notion. We now state our hypotheses and results. We assume $F$ is a Hamilton-JacobiBellman (HJB) operator, that is, a supremum of linear operators like (1.2)

$$
F[u]=F\left(D^{2} u, D u, u, x\right)=\sup _{\alpha \in \mathcal{A}}\left\{L^{\alpha} u(x)\right\},
$$

where the index $\alpha$ varies in some set $\mathcal{A}$ and

(S) for some constants $0<\lambda \leq \Lambda, \gamma \geq 0$, the matrix $A^{\alpha}(x):=\left(a_{i j}^{\alpha}(x)\right)$ is such that $A^{\alpha} \in C(\bar{\Omega}), \lambda I \leq A^{\alpha}(x) \leq \Lambda I$, and $\left|b^{\alpha}(x)\right|,\left|c^{\alpha}(x)\right| \leq \gamma$, for almost all $x \in \Omega$ and all $\alpha \in \mathcal{A}$.

(C) $F$ satisfies the following comparison principle : if $u, v \in C(\bar{\Omega})$ are $L^{N_{-}}$ viscosity solutions of $F[u] \geq F[v]$ in $\Omega$ and one of $u, v$ is in $W_{\text {loc }}^{2, N}(\Omega)$, then $u \leq v$ in $\Omega$.

Notice we do not assume much regularity on the coefficients of $F$, however all our results are new even for operators with smooth coefficients. For detailed description of the theory and the numerous applications of HJB operators, we refer to the book [15] and to the surveys [24], [33] and [3]. In particular, 
it is shown in [26] and [29] that, under (S) the operator $F$ has two real "principal half-eigenvalues" $\lambda_{1}^{+}(F) \leq \lambda_{1}^{-}(F)$ that correspond to a positive and a negative eigenfunction and

$$
F \text { satisfies }(\mathrm{C}) \Longleftrightarrow \lambda_{1}^{+}(F)>0 .
$$

In order to keep the statements simple, we are going to restrict to nonlinearities $f$ which have the same form as in (1.3). This model case is sufficient to expose the ideas needed in order to study fully nonlinear equations with singular nonlinearities. More general results can be obtained by mingling our techniques with already existing ones, for instance for general decreasing nonlinearities like in [10], or for nonlinearities with an added nonlinear perturbation like $\lambda u^{p}, p>0$ (these types of nonlinearities for fully nonlinear equations were considered in [28] and [14]). We could also consider operators with unbounded coefficients and quadratic dependence in the gradient, like in [32] or operators whose coefficients have some singularity on the boundary of $\Omega$, like in [20]. We leave these extensions to future studies.

Furthermore, all results below extend to Isaacs operators, that is, operators like in (1.4), with the supremum replaced by a combination of suprema and infima of linear operators, provided more regularity in $x$ is assumed, and the operator $F$ is proper (that is, decreasing in $u$ ) so that comparison principles are still available. We refer to [22] and [9] for more precise conditions under which comparison is available for Isaacs operators. All our statements and proofs remain almost unchanged in this case, only the spaces $W^{2, p}$ which appear in them are to be replaced by $C^{1, \alpha}$. Here is our existence and uniqueness theorem.

Theorem 1.1 Suppose $F[u]$ is in the form (1.4) and it satisfies $(S)$ and $(C)$. Assume $\mu>0$ and $p \in L^{N}(\Omega)$, further satisfying $p \geq 0$ in $\Omega$ and $p>0$ on a subset of $\Omega$ with positive measure. Then the problem

$$
F[u]+p(x) u^{-\mu}=0, \quad u>0 \quad \text { in } \quad \Omega, \quad u=0 \quad \text { on } \partial \Omega,
$$

has a unique viscosity solution, such that $u \in W_{\mathrm{loc}}^{2, N}(\Omega) \cap C(\bar{\Omega})$.

In order to prove this theorem we use the method of sub- and supersolutions, as developed for viscosity solutions in [8], which we combine with the recent results on existence of eigenvalues and eigenfunctions of fully nonlinear operators in [29]. We construct super- and sub-solutions by solving a fully nonlinear eigenvalue problem with a weight. Uniqueness follows from the comparison principle.

Our second main result concerns the behaviour of the solution of (1.5) near the boundary of $\Omega$. Naturally, in order to give precise results we need to 
put some restrictions on the weight $p$, the most important (and the simplest) situation being when $p$ is bounded away from zero in $\Omega$. In order to parallel previous results in [12] and [18], we are going to assume that $p$ behaves like a power of the distance function.

Theorem 1.2 Under the hypotheses of Theorem 1.1, assume there are constants $c_{1}, c_{2}>0$ such that

$$
c_{1} d^{\alpha}(x) \leq p(x) \leq c_{2} d^{\alpha}(x), \quad \text { for some } \alpha \geq 0,
$$

where $d(x)=d(x, \partial \Omega)$. Then for the solution $u$ of equation (1.5) we have:

(i) If $\mu<1+\alpha$ then $u \in C^{1, \beta}(\bar{\Omega})$, for some $\beta$ which depends only on $\mu, \alpha, \lambda, \Lambda, \gamma, \delta, N$ and $\Omega$.

(ii) If $\mu=1+\alpha$ then $u \in C^{\beta}(\bar{\Omega})$ for all $\beta<1$, and there exist constants $a_{1}, a_{2}, D>0$ such that

$$
a_{1} d(x)(D-\log d(x))^{1 /(1+\mu)} \leq u(x) \leq a_{2} d(x)(D-\log d(x))^{1 /(1+\mu)},
$$

(iii) If $\mu>1+\alpha$ then $u \in C^{\frac{\alpha+2}{1+\mu}}(\bar{\Omega})$ and for some constants $a_{1}, a_{2}>0$

$$
a_{1} d(x)^{(\alpha+2) /(1+\mu)} \leq u(x) \leq a_{2} d(x)^{(\alpha+2) /(1+\mu)}, \quad x \in \Omega .
$$

The most interesting part of this theorem is statement (i), whose proof uses an extension to (1.5) of the celebrated method of Krylov and Safonov [23], that proved global $C^{1, \beta}$-bounds for solutions of linear equations with measurable coefficients, essentially opening up the theory of equations in non-divergence form. More precisely, we are going to build up on a simplified version of this method due to Caffarelli. To prove our regularity theorem we need to get sharp bounds on the solution near the boundary, which are obtained by appropriate comparison with radially symmetric solutions for related extremal equations. See Section 3 for details.

We further mention that as a by-product of our results we provide solutions for the following degenerate parabolic equation:

$$
\begin{gathered}
p(x) v_{t}=v^{\beta} F\left(D^{2} v, D v, v, x\right) \quad \text { in } \quad \Omega \times(0, \infty), \\
v(x, 0)=v_{0}(x) \quad \text { and } \quad v(x, t)=0 \quad \text { on } \quad \partial \Omega \times(0, \infty) .
\end{gathered}
$$

Indeed, if we take $v=u \eta$, where $u$ is a solution of (1.1) and $\eta$ is a solution of $\eta_{t}=-\eta^{\beta+1}$, with $\mu=\beta-1$ and $\beta>0$, then $v$ satisfies the parabolic equation (1.7). This special solution is suitable for sub- and/or super-solutions of the initial value problem (1.7)-(1.8) for a large class of initial conditions $v_{0}$. 


\section{Proof of Theorem 1.1}

\subsection{Preliminaries and a weighted eigenvalue problem}

We begin this section by restating the structural hypotheses we made on $F$ in the introduction. We assume $F: S_{N} \times \mathbb{R}^{N} \times \mathbb{R} \times \Omega \rightarrow \mathbb{R}$ satisfies, with $S=(M, p, u), T=(N, q, v) \in S_{N} \times \mathbb{R}^{N} \times \mathbb{R}$, the following hypotheses:

(H0) $F$ is positively 1-homogeneous : $F(t S, x)=t F(S, x)$ for $t \geq 0$.

(H1) There exist $0<\lambda \leq \Lambda, \gamma \geq 0$ such that for all $S, T$

$$
\begin{array}{r}
\mathcal{M}^{-}(M-N)-\gamma(|p-q|+|u-v|) \leq F(S, x)-F(T, x) \\
\leq \mathcal{M}^{+}(M-N)+\gamma(|p-q|+|u-v|) .
\end{array}
$$

(H2) $-F(T-S, x) \leq F(S, x)-F(T, x) \leq F(S-T, x)$ for all $S, T$.

(H3) The function $F(M, 0,0, x)$ is continuous in $S_{N} \times \bar{\Omega}$.

Here $\mathcal{M}^{-}, \mathcal{M}^{+}$denote the Pucci extremal operators, defined as follows : $\mathcal{M}^{+}(M)=\sup _{A \in \mathcal{S}_{N}^{\lambda, \Lambda}} \operatorname{tr}(A M), \mathcal{M}^{-}(M)=\inf _{A \in \mathcal{S}_{N}^{\lambda, \Lambda}} \operatorname{tr}(A M)$, where $\mathcal{S}_{N}^{\lambda, \Lambda}$ denotes the set of symmetric matrices whose eigenvalues lie in the interval $[\lambda, \Lambda]$. We have the following alternative way of defining $\mathcal{M}^{-}$and $\mathcal{M}^{+}$

$$
\mathcal{M}^{-}(M)=\lambda \sum_{\mu_{j}>0} \mu_{j}+\Lambda \sum_{\mu_{j}<0} \mu_{j} \quad \text { and } \quad \mathcal{M}^{+}(M)=\lambda \sum_{\mu_{j}<0} \mu_{j}+\Lambda \sum_{\mu_{j}>0} \mu_{j},
$$

where $\mu_{1}, \ldots, \mu_{n}$ are the eigenvalues of $M$, see for instance [4].

Hypotheses (H0)-(H1) define an uniformly elliptic Isaacs operator with bounded measurable coefficients. Such an operator is convex, that is, is in the form (1.4) if and only if it satisfies (H2). As we already noticed, in all what follows (H2) could be replaced by an hypothesis on the regularity of $F$ with respect to $x$ and monotonicity of $F$ with respect to $u$, for instance $F$ is $C^{\alpha}$ in $x$ with $\alpha>1 / 2$ and $c^{\alpha} \leq 0$ in (1.4). See the remarks at the end of Section 1 of [9] and the papers quoted there. These hypotheses guarantee the comparison principle (C) holds, with $W^{2, N}$ replaced by $C^{1, \alpha}$ in its statement. Hypothesis (H3) is used to ensure the well-posedness of the Dirichlet problem. As it is well-known, even a linear equation $a_{i j} \partial_{i j} u=0$ can have more than one viscosity solution if its coefficients are discontinuous, see [27].

Next we recall several known results which we use in the sequel. First we give the comparison and existence results from [5], [9], [29], [36]. 
Theorem 2.1 Suppose F satisfies (H0), (H1), (H2) and (H3). Then the operator $\tilde{F}[u]=F[u]-\gamma u$ satisfies $(C)$ and for any $f \in L^{N}(\Omega)$, there exists a unique viscosity solution $u \in W^{2, N}(\Omega)$ of

$$
F\left(D^{2} u, D u, u, x\right)-\gamma u=f \quad \text { in } \Omega, \quad u=0 \quad \text { on } \partial \Omega .
$$

We also recall the following strong maximum principle (Hopf's lemma), which is a consequence from the results in [2] (a simple proof can be found in the appendix of [1]).

Theorem 2.2 Suppose $w \in C(\bar{\Omega})$ is a viscosity solution of

$$
\mathcal{M}^{-}\left(D^{2} w\right)-\gamma|D w|-\gamma w \leq 0 \text { in } \Omega,
$$

and $w \geq 0$ in $\Omega$. Then either $w \equiv 0$ in $\Omega$ or $w>0$ in $\Omega$ and at any point $x_{0} \in \partial \Omega$ at which $w\left(x_{0}\right)=0$ we have $\liminf _{t \searrow 0} \frac{w\left(x_{0}+t \nu\right)-w\left(x_{0}\right)}{t}>0$, where $\nu$ is the interior normal to $\partial \Omega$ at $x_{0}$.

The next theorem is a simple consequence of the compactness result of [9] (Proposition 4.2 in that paper) and the convergence properties of viscosity solutions (see Theorem 3.8 in [5]).

Theorem 2.3 Let $f_{n} \rightarrow f$ in $L^{N}(\Omega)$. Suppose $F$ satisfies $(H 1)$ and $u_{n}$ is a solution of $F\left(D^{2} u_{n}, D u_{n}, u_{n}, x\right)=f_{n}$ in $\Omega, u_{n}=0$ on $\partial \Omega$, such that the sequence $u_{n}$ is bounded in $L^{\infty}(\Omega)$. Then a subsequence of $\left\{u_{n}\right\}$ converges uniformly to a function $u$, which solves $F\left(D^{2} u, D u, u, x\right)=f$ in $\Omega, u=0$ on $\partial \Omega$.

Now we give a proof of the existence of first half-eigenvalues for fully nonlinear elliptic operators with a non-negative weight. More precisely, we consider the eigenvalue problem

$$
F\left(D^{2} u, D u, u, x\right)=-\lambda p(x) u \quad \text { in } \quad \Omega, \quad u=0 \quad \text { on } \quad \partial \Omega
$$

and we prove the following

Theorem 2.4 Assume F satisfies $(H 0)-(H 3)$. Let $p \in L^{N}(\Omega)$ be such that $p(x) \geq 0$ in $\Omega$ and $p(x)>0$ on a subset of $\Omega$ with positive measure. Then there exists a couple $\left(\lambda^{+}, \varphi^{+}\right) \in \mathbb{R} \times W^{2, N}(\Omega)$ of solutions to (2.2), such that $\varphi^{+}>0$ in $\Omega$. 
Proof of Theorem 2.4. We are going to use the Leray-Schauder alternative given in Corollary 1 of of Theorem VIII.1 in [30]. For that purpose we consider the solution $\mathcal{L}(g)$ of the equation

$$
F\left(D^{2} u, D u, u, x\right)-\gamma u=-p(x) g \quad \text { in } \Omega, \quad u=0 \quad \text { on } \partial \Omega,
$$

which is well defined for every $g \in C(\bar{\Omega})$, thanks to Theorem 2.1. Then we define the cone $K=\{u \in C(\bar{\Omega}) \mid u \geq 0$ in $\Omega, u=0$ on $\partial \Omega\}$ and we observe that $\mathcal{L}(g) \in K$ for all $g \in K$. Next, from the hypothesis on the weight $p$, we can choose a smooth $u_{0} \in K \backslash\{0\}$, with compact support, such that $p(x) u_{0}(x)>0$ on a set of non-zero measure. Then, using Theorem 2.2 we can find $M>0$ such that $M \mathcal{L}\left(u_{0}\right) \geq u_{0}$.

Now we are in a position to define the map $T_{\varepsilon}: \mathbb{R}^{+} \times K \rightarrow K$ as $T_{\varepsilon}(\mu, u)=\mu \mathcal{L}(u)+\mu \varepsilon \mathcal{L}\left(u_{0}\right)$, for $\varepsilon>0$. We notice that by Theorem 2.1 and well known compact imbedding theorems, $T_{\varepsilon}$ is a well defined compact operator. Moreover $T_{\varepsilon}(0, u)=0$. Thus, by quoted Leray-Schauder alternative there exists an unbounded connected component $\mathcal{C}_{\varepsilon} \subset \mathbb{R}^{+} \times K$ of solutions to $T_{\varepsilon}(\mu, u)=u$, containing $(0,0)$. We claim that $\mathcal{C}_{\varepsilon} \subset[0, M] \times K$. To verify this, we have that for all $(\mu, u) \in \mathcal{C}_{\varepsilon}$

$$
u=\mu \mathcal{L}(u)+\mu \varepsilon \mathcal{L}\left(u_{0}\right)
$$

and then $u \geq \mu \mathcal{L}(u)$ and $u \geq \mu \varepsilon \mathcal{L}\left(u_{0}\right) \geq \frac{\mu}{M} \varepsilon u_{0}$. If we apply $\mathcal{L}$ again, by Theorem 2.1 we get

$$
\frac{u}{\mu} \geq \mathcal{L}(u) \geq \frac{\mu}{M} \varepsilon \mathcal{L}\left(u_{0}\right) \geq \frac{\mu}{M^{2}} \varepsilon u_{0}
$$

so that $u \geq\left(\frac{\mu}{M}\right)^{2} \varepsilon u_{0}$. Repeating this step we get

$$
u \geq\left(\frac{\mu}{M}\right)^{n} \varepsilon u_{0} \text { for all } n \geq 2,
$$

and we conclude that $\mu \leq M$. This and the fact that $\mathcal{C}_{\varepsilon}$ is unbounded imply that for each $\varepsilon>0$ there exists $\left(\mu_{\varepsilon}, u_{\varepsilon}\right) \in \mathcal{C}_{\varepsilon}$ such that $\left\|u_{\varepsilon}\right\|_{\infty}=1$. Now Theorem 2.3 allows us to pass to the limit as $\varepsilon \rightarrow 0$, to find $\mu^{+} \in[0, M]$ and $\varphi^{+} \geq 0, \varphi^{+} \neq 0$ such that $\varphi^{+}=\mu^{+} \mathcal{L}\left(\varphi^{+}\right)$. From here we also deduce that $\mu^{+}>0$ and by the Theorem 2.2 that $\varphi^{+}>0$. Finally we define $\lambda_{1}^{+}=-\gamma+\mu^{+}$.

Remark. In Theorem 2.4 we have only stated the existence of an eigenvalue associated to a positive eigenfunction, which is the only result we need here. However it can also be proved that this eigenfunction is simple and that there is a negative eigenfunction associated to a second eigenvalue $\lambda^{-} \geq \lambda^{+}$. Actually, nearly all results from [29] can be extended to (2.2).

In all that follows we denote the above eigenvalues with $\lambda^{+}(F, p)$ to emphasize the weight $p$. 


\subsection{Proof of the existence theorem}

In this subsection we are going to prove our existence and uniqueness Theorem 1.1. Recall that we assume $\lambda^{+}(F, 1)>0$, that is, (C) holds. It follows from the definition of $\lambda^{+}(F)$, see [29], that this eigenvalue is monotone in the domain $\Omega$. Hence the first eigenvalue of $F$ is positive in any subdomain of $\Omega$ as well, so the comparison principle is valid in any subdomain of $\Omega$ (including non-smooth ones, as explained in [29], or as can be seen by approximation).

We now state a comparison theorem for super and sub-solutions of our equation and regularized version of it.

Theorem 2.5 (Comparison) Assume $F$ satisfies $(S)$ and $(C)$. Let $\delta \geq 0$ and $u, v \in W_{\text {loc }}^{2, N}(\Omega)$ be respectively a sub-and a super-solution of

$$
H(w):=F\left(D^{2} w, D w, w, x\right)+p(x)(w+\delta)^{-\mu}=0 \quad \text { in } \quad \Omega,
$$

with $u \leq v$ on $\partial \Omega$. Then $u \leq v$ in $\Omega$.

Proof. Suppose by contradiction that $Z=\{x \in \Omega \mid u>v\}$ is not empty. Let $Z^{*}$ be a connected component of $Z$ and $z=v-u$. Then, since $z=0$ on $\partial Z^{*}$ and $p, \mu \geq 0$ in $\Omega$, by (C) we get $z \geq 0$ in $Z^{*}$, a contradiction.

Next we prove the existence and uniqueness of a solution for a regularized version of our problem. We consider the eigenvalue $\lambda_{p}^{+}:=\lambda^{+}(F, p)$ and the associated positive eigenfunction $\varphi^{+}$.

Proposition 2.1 Assume $F$ satisfies $(S)$ and $(C)$. For every $\delta>0$ there exists a unique solution to (2.4), with $u_{\delta}=0$ on $\partial \Omega$. Moreover, there are positive constants $a, b$ and $0<\eta<1$, independent of $\delta$, such that

$$
a \varphi^{+} \leq u \leq b\left(\varphi^{+}\right)^{\eta} \quad \text { in } \quad \Omega .
$$

Proof. Let $\phi^{+}$be the eigenfunction corresponding to $\lambda_{1}^{+}:=\lambda^{+}(F, 1)>0$. We first observe that

$$
H\left(a \varphi^{+}\right)=-p(x)\left(a \lambda_{p}^{+} \varphi^{+}-\left(a \varphi^{+}+\delta\right)^{-\mu}\right) \geq 0,
$$

for sufficiently small $a>0$, independently of $\delta>0$. Thus $v_{1}=a \varphi^{+}$is a sub-solution to (2.4).

Now we take $v_{2}=\left(\phi^{+}\right)^{\eta}+b \phi^{+}$, where $b>0$ is chosen later and $0<\eta<1$ is a fixed constant such that $\eta<2 /(\mu+1)$. Computing $D v_{2}, D^{2} v_{2}$, and using hypotheses $(\mathrm{H} 0)$ and $(\mathrm{H} 1)$ we see that $v_{2}$ satisfies

$$
H\left(v_{2}\right) \leq F\left[\left(\phi^{+}\right)^{\eta}\right]+b F\left[\phi^{+}\right]+p(x)\left(v_{2}+\delta\right)^{-\mu}
$$




$$
\begin{aligned}
\leq & \eta\left(\phi^{+}\right)^{\eta-1} F\left(D^{2} \phi^{+}, D \phi^{+}, \phi^{+}, x\right)+(1-\eta) \gamma\left(\phi^{+}\right)^{\eta} \\
& +(\eta-1) \eta\left(\phi^{+}\right)^{\eta-2} \mathcal{M}_{\lambda, \Lambda}^{-}\left(D \phi^{+} \otimes D \phi^{+}\right) \\
& +b F\left(D^{2} \phi^{+}, D \phi^{+}, \phi^{+}, x\right)+p(x)\left(v_{2}+\delta\right)^{-\mu} \\
\leq & \left(\phi^{+}\right)^{\eta}\left\{-\lambda_{1}^{+} \eta+(1-\eta) \gamma\right\}-b \lambda_{1}^{+} \phi^{+} \\
& +(\eta-1) \eta\left(\phi^{+}\right)^{\eta-2} \mathcal{M}_{\lambda, \Lambda}^{-}\left(D \phi^{+} \otimes D \phi^{+}\right)+p(x)\left(\phi^{+}\right)^{-\eta \mu} .
\end{aligned}
$$

Notice that the matrix $D \phi^{+} \otimes D \phi^{+}$has $\left|D \phi^{+}\right|^{2}$ as the only nontrivial eigenvalue. By using Theorem 2.2 we see that there exists a neighborhood $V$ of $\partial \Omega$ such that $\left|D \phi^{+}\right| \geq c_{0}>0$ in $V$, that is, for some $k>0$

$$
\eta\left(\phi^{+}\right)^{\eta-2} \mathcal{M}_{\lambda, \Lambda}^{-}\left(D \phi^{+} \otimes D \phi^{+}\right) \geq k\left(\phi^{+}\right)^{\eta-2},
$$

in $V$. Now, recalling that $\eta-1<0$ and $2-\eta>\mu \eta$, by the fact that $\phi^{+}$ vanishes on $\partial \Omega$ and the inequalities (2.6) and (2.7), we find that for large $b$ the inequality $H\left(v_{2}\right) \leq 0$ holds in $V$. On the other hand, in $\Omega \backslash V$ we find that $H\left(v_{2}\right) \leq 0$, again by choosing $b$ large enough.

Using $v_{1}$ and $v_{2}$ as a sub- and a super-solution for (2.4), we may now use the standard method of monotone iterations, to find a solution $u_{\delta}$ for $(2.4)$, which vanishes on $\partial \Omega$. By the regularity result of [35], $u_{\delta}$ is in $W_{\text {loc }}^{2, N}(\Omega)$. Moreover, by enlarging $b$ we find that $u_{\delta} \leq b\left(\varphi^{+}\right)^{\eta}$ in $\Omega$, so that (2.5) also holds.

Remark. In the proof of this proposition we need that $\lambda^{+}(F, 1)>0$ and that the weighted eigenvalue problem has a solution, but we do not use the sign of $\lambda^{+}(F, p)$.

We end this section with a proof of Theorem 1.1, the existence and uniqueness result for the singular equation.

Proof of Theorem 1.1. Take a sequence $\delta_{n}=1 / n$ and use Proposition 2.1 to find a monotone sequence of solutions $u_{n}=u_{\delta_{n}}$ to equation (2.4) satisfying (2.5) in $\Omega$. Then, by Theorem 2.3 and a standard diagonal procedure, we find a solution $u$ to (1.1). Uniqueness follows from Theorem 2.5.

\section{Estimates on the solution near the bound- ary of the domain}

The first goal of this section is to obtain a sharp bound for the solutions to (1.1) near the boundary of $\Omega$, under some extra assumptions on $p$. Precisely, we prove the following theorem: 
Theorem 3.1 Assume $F$ satisfies $(S)$ and $(C)$. Suppose there are constants $c_{1}, c_{2}>0$ such that

$$
c_{1} d^{\alpha}(x) \leq p(x) \leq c_{2} d^{\alpha}(x), \quad \alpha \geq 0,
$$

where $d(x)=d(x, \partial \Omega)$. Let $u$ be the solution to (1.1). Then

(i) If $\mu<1+\alpha$ then there exist constants $a_{1}, a_{2}>0$ such that

$$
a_{1} d(x) \leq u(x) \leq a_{2} d(x), \quad x \in \Omega .
$$

(ii) If $\mu=1+\alpha$ then there exist constants $a_{1}, a_{2}, D>0$ such that

$$
a_{1} d(x)(D-\log d(x))^{1 /(1+\mu)} \leq u(x) \leq a_{2} d(x)(D-\log d(x))^{1 /(1+\mu)} .
$$

(iii) If $\mu>1+\alpha$ then there exist constants $a_{1}, a_{2}>0$ such that

$$
a_{1} d(x)^{(\alpha+2) /(1+\mu)} \leq u(x) \leq a_{2} d(x)^{(\alpha+2) /(1+\mu)}, \quad x \in \Omega .
$$

In what follows we consider the following two operators

$$
F^{ \pm}(u)=\mathcal{M}_{\lambda, \Lambda}^{ \pm}\left(D^{2} u\right) \pm \gamma|D u| \pm \gamma u,
$$

which are the extremal operators appearing in hypothesis (H1).

Let us denote by $B_{r}$ the ball with radius $r$ centered at the origin, and set $A_{\rho, R}=\{x \mid \rho<r<R\}$, for $0<\rho<R$ (we denote $r=|x|$ ). In our first lemma we construct an appropriate comparison function, to be used as supersolution for (1.1) near the boundary of $\Omega$. Note that this construction can only be done in a small neighbourhood of the boundary, since $F^{+}$contains a positive zero order term, and is therefore not coercive (that is, its first eigenvalue is not positive) in large domains.

Lemma 3.1 Assume $\alpha \geq 0, \mu>0$. There exists $R_{0}>0$ such that for every $0<\rho<R \leq R_{0}, C>0$ and $M>0$, the problem

$$
\begin{gathered}
F^{+}(v)+C(r-\rho)^{\alpha} v^{-\mu}=0 \quad \text { in } \quad A_{\rho, R}, \\
v=0 \text { on } \partial B_{\rho}, \quad v=M \text { on } \partial B_{R},
\end{gathered}
$$

has a unique positive solution.

Proof. Since $F^{+}$satisfies (H0)-(H3), by Theorem 2.4 there exist $\varphi^{+}>0$ and $\lambda^{+} \in \mathbb{R}$, such that

$$
F^{+}\left(\varphi^{+}\right)+\lambda^{+}(r-\rho)^{\alpha} \varphi^{+}=0 \text { in } A_{\rho, R}, \quad \varphi^{+}=0 \text { on } \partial A_{\rho, R} .
$$


We also have an eigenpair $\left(\lambda_{1}^{+}, \phi^{+}\right)$which solves

$$
F^{+}\left(\phi^{+}\right)+\lambda_{1}^{+} \phi^{+}=0 \text { in } A_{\rho, R}, \quad \phi^{+}=0 \text { on } \partial A_{\rho, R},
$$

so, in particular

$$
\mathcal{M}_{\lambda, \Lambda}^{+}\left(D^{2} \phi^{+}\right)+\gamma\left|D \phi^{+}\right| \geq\left(-\lambda_{1}^{+}-\gamma\right) \phi^{+} \text {in } A_{\rho, R}, \quad \phi^{+}=0 \text { on } \partial A_{\rho, R} .
$$

Applying the ABP inequality (see [5]) to $\phi^{+}$we obtain

$$
\sup _{\Omega} \phi^{+} \leq C \operatorname{diam}\left(A_{\rho, R}\right)\left(\lambda_{1}^{+}+\gamma\right) \sup _{\Omega} \phi^{+}
$$

So

$$
\lambda_{1}^{+} \geq \frac{C}{R-\rho}-\gamma
$$

Hence if we choose $R_{0}$ small enough we have $\lambda_{1}^{+}>0$. Thus, proceeding as in the proof of Proposition 2.1 and that of Theorem 1.1, we only need to find appropriate sub and super solutions for

$$
\begin{array}{r}
F^{+}(v)+C(r-\rho)^{\alpha}(v+\delta)^{-\mu}=0, \quad \text { in } A_{\rho, R}, \\
v=0 \text { on } \partial B_{\rho}, \quad v=M \text { on } \partial B_{R},
\end{array}
$$

which are independent of $\delta>0$. If we consider $a$ small enough then $v_{1}=a \varphi^{+}$, is a sub-solution for (3.3)-(3.4), as we proved in Pr2.1. We observe that $v_{1}(\rho)=0$ and $v_{1}(R)=0<M$.

Next we find a super-solution for (3.3)-(3.4). As in Proposition 2.1, we consider a constant $0<\eta<1$ such that $\eta<2 /(\mu+1)$. Let us prove that $v_{2}=\left(\phi^{+}\right)^{\eta}+b \phi^{+}+\bar{v}$ is a super-solution, with $\bar{v}:=M(r-\rho) /(R-\rho)$. In fact, if we define for a function $w$

$$
H(w)=F^{+}(w)+C(r-\rho)^{\alpha}(w+\delta)^{-\mu},
$$

we easily check that

$$
\begin{aligned}
H\left(v_{2}\right) \leq & F^{+}\left(\left(\phi^{+}\right)^{\eta}\right)+b F^{+}\left(\phi^{+}\right)+F^{+}(\bar{v})+C(r-\rho)^{\alpha}\left(\phi^{+}\right)^{-\eta \mu} \\
\leq & \gamma_{2}(1-\eta)\left(\phi^{+}\right)^{\eta}-b \lambda_{1}^{+} \phi^{+}+F^{+}(\bar{v}) \\
& +\eta(\eta-1) \lambda\left(\phi^{+}\right)^{\eta-2}\left|\left(\phi^{+}\right)^{\prime}\right|^{2}+C(r-\rho)^{\alpha}\left(\phi^{+}\right)^{-\eta \mu}
\end{aligned}
$$

Recalling that $\eta-2<-\eta \mu<0$ and $\alpha \geq 0$, since we have $\left|\left(\phi^{+}\right)^{\prime}(R)\right|>0$, $\left|\left(\phi^{+}\right)^{\prime}(\rho)\right|>0, \phi^{+}(\rho)=F^{+}(\bar{v})(\rho)=0$ and $F^{+}(\bar{v}) \leq C$, for a certain constant $C$, we see that the right hand side in (3.7) is negative near $r=\rho$ and $r=R$. Then we choose $b$ large enough so that $H\left(v_{2}\right) \leq 0$ in the whole interval, 
completing the proof that $v_{2}$ is a super-solution. We notice that $v_{2}$ satisfy the boundary conditions (3.4).

Now we take $\lambda_{0}$ large enough so that the function

$$
G(s, r)=-C(r-\rho)^{\alpha}(s+\bar{v}(r)+\delta)^{-\mu}-\lambda_{0} s
$$

is decreasing in $s$ for all $r \in(\rho, R)$. Then by the usual iteration procedure we can solve the hierarchy of equations

$F\left(D^{2} w_{i+1}+D^{2} \bar{v}, D w_{i+1}+D \bar{v}, w_{i+1}+\bar{v}, r\right)-\lambda_{0} w_{i+1}=G\left(w_{i}, r\right), \quad r \in(\rho, R)$,

with Dirichlet boundary conditions $w_{i+1}(\rho)=w_{i+1}(R)=0$. The sequence $v_{i}=w_{i}+\bar{v}$ is decreasing and bounded, so convergent to a solution of (3.5)(3.6). By letting $\delta \rightarrow 0$ we then get a solution to our problem.

\section{Proof of Theorem 3.1.}

Proof of statement (i). We first consider the case $\alpha-\mu>-1$. We start by analyzing the behavior of the radial solution $u$ of (3.3)-(3.4), found in Lemma 3.1. We will prove that there is a positive constant $a_{2}$ such that

$$
u(r) \leq a_{2}(r-\rho), \quad \text { for all } \quad r \in[\rho, R] .
$$

We will assume, without loss of generality, that $R \leq 1$. It is convenient to define the function $\theta(s)$ by $\theta(s)=\Lambda$ if $s \geq 0$, and $\theta(s)=\lambda$ if $s<0$. Then, since $u$ is radially symmetric, the eigenvalues of $D^{2} u$ are $u^{\prime \prime}(r)$ and $u^{\prime}(r) / r$, so by the properties of $\mathcal{M}^{+}$we have

$$
\mathcal{M}^{+}\left(D^{2} u\right)(r)=\theta\left(u^{\prime \prime}(r)\right) u^{\prime \prime}(r)+\theta\left(u^{\prime}(r)\right)(N-1) \frac{u^{\prime}(r)}{r} .
$$

Thus $u$ satisfies the equation

$$
\theta\left(u^{\prime \prime}(r)\right) u^{\prime \prime}+\theta\left(u^{\prime}(r)\right)(N-1) \frac{u^{\prime}}{r}+\gamma\left|u^{\prime}\right|+\gamma u+C(r-\rho)^{\alpha} u^{-\mu}=0 .
$$

In order to write this equation in a simpler form, we define

$$
\begin{gathered}
\nu(r)=\frac{\theta\left(u^{\prime}(r)\right)(N-1)}{\theta\left(u^{\prime \prime}(r)\right) r} \\
\zeta(r)=\exp \left(\int_{1}^{r} \nu(s) d s\right) \text { and } \tilde{\zeta}(r)=\frac{\zeta(r)}{\theta\left(u^{\prime \prime}(r)\right)} .
\end{gathered}
$$

Then (3.9) can be written as

$$
\left(\zeta u^{\prime}\right)^{\prime}+\tilde{\zeta}\left\{-\gamma\left|u^{\prime}\right|-\gamma u+C(r-\rho)^{\alpha} u^{-\mu}\right\}=0 .
$$


If we set $N_{+}=\frac{\lambda}{\Lambda}(N-1)+1$, and $N_{-}=\frac{\Lambda}{\lambda}(N-1)+1$, then we easily see that for all $\rho \leq r \leq R \leq 1$ we have

$$
\begin{gathered}
N_{+}-1 \leq \nu(r) r \leq N_{-}-1 \\
r^{N_{-}-1} \leq \zeta(r) \leq r^{N_{+}-1} \text { and } \frac{\zeta(r)}{\Lambda} \leq \tilde{\zeta}(r) \leq \frac{\zeta(r)}{\lambda} .
\end{gathered}
$$

Let $r_{0}=\sup \left\{r \in[\rho, R] \mid u^{\prime}(s)>0, s \leq r\right\}$. By Hopf's lemma $r_{0}>\rho$. Integrating for $r \in\left(\rho, r_{0}\right)$, we find

$$
\begin{aligned}
u^{\prime}(r) & \leq(\zeta(r))^{-1} \int_{r}^{r_{0}} \tilde{\zeta}(s)\left[C a_{1}^{-\mu}(s-\rho)^{\alpha-\mu}+\gamma u^{\prime}(s)+\gamma u(s)\right] d s \\
& \leq C\left\{1+\frac{1}{\xi(r)} \int_{r}^{r_{0}} u^{\prime}(s) \xi(s) d s+\int_{r}^{r_{0}}(s-\rho)^{\alpha-\mu} d s\right\}<\infty
\end{aligned}
$$

since $\alpha-\mu>-1,0<c(\rho) \leq \xi(r), \tilde{\xi}(r) \leq C$, and $u$ is bounded. Thus $u^{\prime}(r)$ is bounded for $r \in\left[\rho, r_{0}\right]$, from which we deduce the existence of a constant $a_{2}$ such that $u(r) \leq a_{2}(r-\rho)$ for all $r \in[\rho, R]$, completing the proof of (3.8).

Now we prove Theorem 3.1 (i). Since $\Omega$ is smooth and bounded we can find $\rho>0$ such that for every point $x_{0} \in \Omega$ such that $d\left(x_{0}\right)<\rho$, there exist points $y_{0}=y\left(x_{0}\right) \in \partial \Omega$ and $z_{0}=z\left(x_{0}\right) \notin \Omega$ along the normal direction at $y_{0}$, with $\left|y_{0}-z_{0}\right|=\rho$. Set $R=2 \rho$ and decrease $\rho$, if necessary, to have $2 \rho<R_{0}$, where $R_{0}$ was given in Lemma 3.1. Note that $B\left(z_{0}, \rho\right)$ is an exterior tangent ball to $\partial \Omega$.

Using the hypothesis (3.1) we see that for all $x \in \Omega \cap B\left(z_{0}, R\right)$

$$
p(x) \leq c_{2} d^{\alpha}(x) \leq c_{2}\left(\left|x-z_{0}\right|-\rho\right)^{\alpha} .
$$

Now we consider the solution $u$ of the singular equation (1.1), given by Theorem 1.1, together with the solution $v$ of (3.3)-(3.4) with $C \geq c_{2}$ and $M=\sup _{x \in \Omega} u(x)$. By using Theorem 2.5 we conclude that $u(x) \leq v(x)$ for all $x \in B\left(z_{0}, R\right) \backslash B\left(z_{0}, \rho\right)$. Finally, using (3.8) we get that

$$
u\left(x_{0}\right) \leq v\left(x_{0}\right)=v\left(\left|x_{0}-z_{0}\right|\right) \leq a_{2}\left(\left|x_{0}-z_{0}\right|-\rho\right)=a_{2} d\left(x_{0}\right) .
$$

Since $x_{0}$ is arbitrary among the points $x \in\{x \in \Omega \mid d(x)<\rho\}$ we obtain the desired upper estimate. The lower estimate is given in the proof of Theorem 1.1 , thus case (i) is complete.

Remark. Note that only the inequality $p(x) \leq d^{\alpha}(x)$ was needed for this proof.

Proof of statement (ii). This case can be treated by giving explicit super and sub-solutions as in [18]. Let us construct a super-solution, in order to obtain 
the upper estimate. As above, we consider $\rho$ and $R=(1+\sigma) \rho$, with $\rho$ small enough and $\sigma>0$ to be fixed later. We further assume $R=(1+\sigma) \rho<1$. We define

$$
u_{2}(r)=\bar{C}(r-\rho)(D-\log (r-\rho))^{1 /(1+\mu)},
$$

where $D>1+\log (2 \operatorname{diam}(\Omega))$ and $\bar{C}$ are chosen later. Setting

$$
h(r)=(D-\log (r-\rho))^{-\mu /(1+\mu)},
$$

a simple computation shows that

$$
\begin{gathered}
u_{2}^{\prime}(r)=\bar{C} h(r)(D-\log (r-\rho)-1 /(1+\mu)) \\
\left.u_{2}^{\prime \prime}(r)=\frac{-\bar{C}}{(1+\mu)(r-\rho)} h(r)\left\{1+\frac{\mu}{1+\mu}(D-\log (r-\rho))^{-1}\right)\right\}, \\
\frac{c_{2}(r-\rho)^{\alpha}}{u_{2}^{\mu}}=\frac{c_{2} h(r)}{\bar{C}^{\mu}(r-\rho)},
\end{gathered}
$$

where we used the assumption $\mu=\alpha+1$. Next we observe that $u_{2}^{\prime}(r)>0$, $u_{2}^{\prime \prime}(r)<0$, and we claim that $\sigma$ can be chosen to have

$$
\begin{aligned}
\mathcal{M}_{\lambda, \Lambda}^{+}\left(D^{2} u_{2}\right) & =\lambda u_{2}^{\prime \prime}+\Lambda(N-1) \frac{u_{2}^{\prime}}{r} \\
& \leq-\frac{\bar{C} \lambda h(r)}{2(1+\mu)}(r-\rho)^{-1}
\end{aligned}
$$

for all $r \in(\rho, R)$. In fact, we compute

$$
\mathcal{M}_{\lambda, \Lambda}^{+}\left(D^{2} u_{2}\right)=\bar{C}(r-\rho)^{-1} h(r) A_{\lambda, \Lambda}(r),
$$

where $A_{\lambda, \Lambda}(r)$ denotes the following expression

$$
\frac{\Lambda(N-1)(r-\rho)}{r}\left(D-\frac{1}{1+\mu}-\log (r-\rho)\right)-\frac{\lambda}{1+\mu}\left(1+\frac{\mu}{1+\mu}(D-\log (r-\rho))^{-1}\right) .
$$

Then we see that for every $r \in(\rho, R)$

$$
A_{\lambda, \Lambda}(r) \leq-\frac{\lambda}{1+\mu}+\Lambda(N-1) \sigma\left(D-\frac{1}{1+\mu}-\log \sigma\right),
$$

from which we infer that $\sigma$ can be chosen small enough to verify the claim. Then, from (3.2), (3.16) and the explicit formulae (3.12)-(3.15) we easily see that by choosing $\bar{C}$ large and $\sigma$ small we obtain

$$
F^{+}\left(u_{2}\right)+\frac{c_{2}(r-\rho)^{\alpha}}{u_{2}^{\mu}} \leq-\frac{\bar{C} h(r)}{r-\rho}\left(1+k_{1}(r-\rho) \log (r-\rho)-\frac{k_{2}}{\bar{C}^{\mu+1}}\right) \leq 0 .
$$


We may choose $\bar{C}$ even larger so that $u_{2}(R) \geq M$, where $M=\sup _{x \in \Omega} u(x)$. As before we use Theorem 2.5 and obtain

$$
u\left(x_{0}\right) \leq u_{2}\left(x_{0}\right)=c d\left(x_{0}\right)\left(D-\log \left(d\left(x_{0}\right)\right)\right)^{1 /(1+\mu)}
$$

for all $x_{0} \in\{x \in \Omega \mid d(x)<\rho\}$. By enlarging $D$, if necessary, we see that this inequality holds for all $x_{0} \in \Omega$, as desired.

To obtain the lower bound the argument is symmetric. We consider $\rho$ small enough and

$$
u_{1}(r)=\bar{c}(\rho-r)(D-\log (\rho-r))^{1 /(1+\mu)}
$$

where $D$ and $\bar{c}$ are chosen later. From the definition of $F^{-}$given in (3.2) and the formulae (3.13)-(3.15) we easily see that by choosing $\bar{c}$ small enough we obtain for $r \in(\rho / 2, \rho)$

$$
F^{-}\left(u_{1}\right)+\frac{c_{1}(\rho-r)^{\alpha}}{u_{1}^{\mu}} \geq \frac{\bar{c} h(r)}{r-\rho}\left(-A_{\Lambda, \lambda}(r)-k_{1}(r-\rho) \log (r-\rho)+\frac{k_{2}}{\bar{c}^{\mu+1}}\right),
$$

which is positive for small $\bar{c}>0$, since $A_{\Lambda, \lambda}(r) \leq$ const. We decrease $\bar{c}$, if necessary, so that $u_{1}(\rho / 2) \leq m$, where

$$
m=\min \{u(x) \mid d(x) \geq \rho / 2\} .
$$

Now, given $x_{0} \in \Omega$ such that $d\left(x_{0}\right)<\rho / 2$, there are points $y_{0}=y\left(x_{0}\right) \in \partial \Omega$ and $z_{0}=z\left(x_{0}\right) \in \Omega$ such that $\left|y_{0}-z_{0}\right|=\rho$ and $d\left(x_{0}\right)=\rho-\left|x_{0}-z_{0}\right|$. Now $B\left(z_{0}, \rho\right)$ is an interior tangent ball to $\partial \Omega$. Then we again use Theorem 2.5 in the annulus $B\left(z_{0}, \rho\right) \backslash B\left(z_{0}, \rho / 2\right)$, to obtain in particular that

$$
u\left(x_{0}\right) \geq u_{1}\left(x_{0}\right)=c d\left(x_{0}\right)\left(D-\log \left(d\left(x_{0}\right)\right)\right)^{1 /(1+\mu)} .
$$

This inequality holds for all $x_{0} \in\{x \in \Omega / d(x)<\rho / 2\}$. By decreasing $c$ even more, if necessary, we see that this inequality holds for all $x_{0} \in \Omega$, as desired. Proof of statement (iii). Finally we study the case $\alpha+1<\mu$. We take again $\rho$ small and $R=(1+\sigma) \rho$, with $\sigma$ to be chosen later. We consider

$$
\begin{gathered}
u_{2}(r)=\bar{C}(r-\rho)^{(2+\alpha) /(1+\mu)}, \quad u_{2}^{\prime}(r)=\frac{\bar{C}(2+\alpha)}{1+\mu}(r-\rho) h(r), \\
u_{2}^{\prime \prime}(r)=\frac{\bar{C}(2+\alpha)(1+\alpha-\mu)}{(1+\mu)^{2}} h(r) \quad \text { and } \\
\frac{c_{2}(\rho-r)^{\alpha}}{u_{2}^{\mu}}=\frac{c_{2} h(r)}{\bar{C}^{\mu}}, \quad \text { with } \quad h(r)=(r-\rho)^{(2+\alpha) /(1+\mu)-2} .
\end{gathered}
$$


Then we claim that, if we choose $\sigma$ small enough, we have

$$
\mathcal{M}_{\lambda, \Lambda}^{+}\left(D^{2} u_{2}\right) \leq \bar{C} \lambda \frac{(2+\alpha)(1+\alpha-\mu)}{2(1+\mu)^{2}} h(r)
$$

for all $r \in(\rho, R)$. We compute and we find

$$
\mathcal{M}_{\lambda, \Lambda}^{+}\left(D^{2} u_{2}\right)=\frac{(2+\alpha) h(r)}{1+\mu}\left(\frac{\lambda(1+\alpha-\mu)}{1+\mu}+\frac{\Lambda(N-1)(r-\rho)}{r}\right) .
$$

Since $(r-\rho) / r \leq \sigma$, for all $r \in(r, R)$, by choosing $\sigma$ small enough, the claim follows. Now, taking into account the formulae obtained above we easily see that, by choosing $c$ large enough we we obtain

$$
F^{+}\left(u_{2}\right)+\frac{c_{2}(r-\rho)^{\alpha}}{u_{2}^{\mu}} \leq 0 .
$$

Then we continue with the comparison exactly as in case (ii).

In order to obtain the lower bound, we proceed as in case (ii), but taking as sub-solution $u_{1}(r)=\bar{c}(r-\rho)^{(2+\alpha) /(1+\mu)}$. This completes the proof of Theorem 3.1.

\section{Global regularity of the solution in $\bar{\Omega}$}

In this section we prove Theorem 1.2, that is, we obtain regularity results in the whole domain $\bar{\Omega}$. We notice that interior regularity of the solutions can be obtained by the general theory as in [35]. However, due to the singularity of the equation at $u=0$, that is, on the boundary of the domain, global regularity requires additional arguments which we provide in this section. We recall that we have already obtained bounds on the solutions in Theorem 3.1 .

Our first theorem deals with the case of a strong singularity which occurs when $\alpha-\mu+1 \leq 0$.

Theorem 4.1 Assume $F$ satisfies $(S)$ and $(C)$.

(i) Assume $\alpha+1<\mu$ and (3.1) holds. Then the solution $u$ of (1.1) is in $C^{\frac{\alpha+2}{1+\mu}}(\bar{\Omega})$.

(ii) Assume $\alpha+1=\mu$ and (3.1) holds. Then the solution $u$ of (1.1) is in $C^{\beta}(\bar{\Omega})$, for all $\beta<1$.

Proof. The first step is to straighten the boundary, which is assumed at least $C^{2}$-smooth. This can be done easily by using the computation in the 
proof of Lemma 6.5 in [16] and the representation of $F$ as a supremum (or a sup-inf) of linear operators. With the change of variables each linear operator is modified, but the resulting supremum satisfies (H0)-(H3), possibly with modified constants. More precisely, if $x_{0}$ is fixed point on $\partial \Omega$ there exists a neighbourhood $A_{x_{0}}$ and a $C^{2}$-diffeomorphism $y=\Psi_{0}(x)$ such that $\Psi_{0}\left(A_{x_{0}} \cap \partial \Omega\right)$ is a hyperplane portion of the boundary of $\Psi_{0}\left(A_{x_{0}} \cap \Omega\right)$, say a portion of $\left\{y_{N}=0\right\}$. From now on we assume $u$ is a viscosity solution in $C^{0}\left(\bar{B}^{+}\right)$of

$$
F\left(D^{2} u, D u, u, x\right)=g(x) \quad \text { in } \quad B^{+}, \quad u=0 \quad \text { on } T,
$$

where $B^{+}:=B_{R_{0}} \cap \mathbb{R}_{+}^{N}$ and $T=B_{R_{0}} \cap \partial \mathbb{R}_{+}^{N}$, for some ball $B_{R_{0}}$ of small radius $R_{0}$.

We first consider the case $\alpha-\mu+1<0$. By (H1) and Theorem 3.1 we know that the solution of (1.5) satisfies (4.1), with a function $g$ such that for some $a>0$

$$
\left|g\left(x^{\prime}, x_{N}\right)\right| \leq a x_{N}^{(\alpha-2 \mu) /(\mu+1)} \quad \text { in } \quad B^{+} .
$$

Let $0<R_{2}<R_{1}<R_{0}$ and $T_{2}=B_{R_{2}} \cap \partial \mathbb{R}_{+}^{N}$. There is $\rho_{0}>0$ so that $\left(x^{\prime}, 6 \rho_{0}\right) \in B_{R_{1}}^{+}$for all $\left(x^{\prime}, 0\right) \in T_{2}$. Then fix $\left(\bar{x}^{\prime}, 0\right) \in T_{2}$, define $x_{\rho}=\left(\bar{x}^{\prime}, 3 \rho\right)$, for $0<\rho<\rho_{0}$ and the scaled function

$$
w(y)=\frac{u\left(\rho y+x_{\rho}\right)}{\rho^{\tau_{1}}}, \quad y \in B_{3}
$$

where $\tau_{1}:=(2+\alpha) /(1+\mu)$. By Theorem $3.1 w$ is bounded independently of $\rho$ in $B_{3}$, and satisfies

$$
F_{\rho}[w]:=F\left(D^{2} w, \rho D w, \rho^{2} w, \rho y+x_{\rho}\right)=g_{\rho}(y) \quad \text { in } B_{3},
$$

where $g_{\rho}(y)=g\left(\rho y+x_{\rho}\right) \rho^{2-\tau_{1}}$. By (4.2) and our choice of $\tau_{1}$ we see that $\left|g_{\rho}(y)\right| \leq c$ for all $y \in B_{2}$, with $c$ independent of $\rho$. Observe that $F_{\rho}$ satisfies (H0)-(H3), since $\rho \leq 1$. Then we use the interior elliptic estimates for this problem, as in [35], to deduce that $w \in C^{1}\left(\bar{B}_{2}\right)$. Thus there exists a constant $C$ independent of $\rho$, such that

$$
\left|w\left(y_{1}\right)-w\left(y_{2}\right)\right| \leq C\left|y_{1}-y_{2}\right|, \quad \text { for all } \quad y_{1}, y_{2} \in B_{2} \text {. }
$$

From this we infer that

$$
\left|u\left(x_{1}\right)-u\left(x_{2}\right)\right| \leq C\left|x_{1}-x_{2}\right|^{\tau_{1}}, \quad \text { for all } y_{1}, y_{2} \in B\left(x_{\rho}, \rho\right),
$$

where the estimate is uniform in $\rho \in\left(0, \rho_{0}\right)$ and $\bar{x}^{\prime} \in T_{2}$. 
Next we claim that for a given $\bar{x}^{\prime}$ the one-dimensional function $z(s)=$ $u\left(\bar{x}^{\prime}, s\right)$ satisfies

$$
\left|z\left(s_{1}\right)-z\left(s_{2}\right)\right| \leq C\left|s_{1}-s_{2}\right|^{\tau_{1}}, \quad \text { for all } s_{1}, s_{2} \in\left[0,4 \rho_{0}\right),
$$

where the constant $C$ can be chosen independently of $\bar{x}^{\prime} \in T_{2}$. To prove the claim we define the sequence $\rho_{i}=(1 / 2)^{i} \rho_{0}$ for $i \in \mathbb{N}$. We assume first that $s_{1}>s_{2}>0$ and later consider the case $s_{2}=0$. Let $i \leq j$ be indices such that $s_{1} \in\left[2 \rho_{i+1}, 2 \rho_{i}\right]$ and $s_{2} \in\left[2 \rho_{j+1}, 2 \rho_{j}\right]$. If $i=j$ then $\left(\bar{x}^{\prime}, s_{1}\right),\left(\bar{x}^{\prime}, s_{2}\right) \in$ $B\left(x_{\rho_{i+1}}, \rho_{i+1}\right)$ and (4.5) holds, by (4.4). If $j=i+1$ then

$$
\frac{\left|z\left(s_{1}\right)-z\left(s_{2}\right)\right|}{\left|s_{1}-s_{2}\right|^{\gamma}} \leq \frac{\left|z\left(s_{1}\right)-z\left(2 \rho_{i+1}\right)\right|}{\left|s_{1}-s_{2}\right|^{\gamma}}+\frac{\left|z\left(2 \rho_{j}\right)-z\left(s_{2}\right)\right|}{\left|s_{1}-s_{2}\right|^{\gamma}} \leq 2 C,
$$

since $s_{1}-s_{2} \geq s_{1}-2 \rho_{i+1}$ and $s_{1}-s_{2} \geq 2 \rho_{j}-s_{2}$. If $i<j+1$ then we have

$$
\begin{aligned}
\frac{\left|z\left(s_{1}\right)-z\left(s_{2}\right)\right|}{\left|s_{1}-s_{2}\right|^{\gamma} \leq} & \frac{\left|z\left(s_{1}\right)-z\left(2 \rho_{i+1}\right)\right|}{\left|s_{1}-s_{2}\right|^{\gamma}}+\sum_{k=i+1}^{k=j-1}\left\{\frac{\left|z\left(2 \rho_{k}\right)-z\left(2 \rho_{k+1}\right)\right|}{\left|s_{1}-s_{2}\right|^{\gamma}}\right\} \\
& +\frac{\left|z\left(2 \rho_{j}\right)-z\left(s_{2}\right)\right|}{\left|s_{1}-s_{2}\right|^{\gamma}} \\
\leq & 2 C+C \sum_{k=i+1}^{k=j-1}\left(\frac{1}{2}\right)^{\gamma(k-i-1)} \leq k C
\end{aligned}
$$

where $k>0$ independent of $i, j$ since the series converges, proving the claim in case $s_{2}>0$. Here we used that $s_{1}-s_{2} \geq(1 / 2)^{i+1} \rho_{0}=(1 / 2)^{i+1-k}\left(2 \rho_{k}-2 \rho_{k+1}\right)$ In the case $s_{2}=0$, we use the continuity of $u$ to obtain that

$$
z\left(s_{1}\right)-z(0)=z\left(s_{1}\right)-z\left(2 \rho_{i+1}\right)+\sum_{k=i+1}^{k=\infty}\left\{z\left(2 \rho_{k}\right)-z\left(2 \rho_{k+1}\right)\right\},
$$

from where we proceed as before, completing the proof of the claim.

Then we prove Hölder continuity of $u$ in all $\bar{B}_{R_{2}}^{+}$as follows. Given $x=$ $\left(x^{\prime}, x_{N}\right), y=\left(y^{\prime}, y_{N}\right) \in \bar{B}_{R_{2}}^{+}$we consider two cases:

i) If we have $|x-y|<x_{N} / 3$ (or $|x-y|<y_{N} / 3$ ) we just apply (4.4) in a ball containing both $x$ and $y$.

ii) Otherwise we have

$$
\begin{aligned}
\frac{\left|u(x)-u\left(x^{\prime}, 0\right)\right|}{|x-y|^{\gamma}} \leq 3^{\gamma} \frac{\left|u(x)-u\left(x^{\prime}, 0\right)\right|}{x_{N}^{\gamma}} \leq 3^{\gamma} C, \\
\frac{\left|u(y)-u\left(y^{\prime}, 0\right)\right|}{|x-y|^{\gamma}} \leq 3^{\gamma} \frac{\left|u(y)-u\left(y^{\prime}, 0\right)\right|}{x_{N}^{\gamma}} \leq 3^{\gamma} C,
\end{aligned}
$$


by using (4.5). Since $u\left(x^{\prime}, 0\right)=u\left(y^{\prime}, 0\right)=0$, the Hölder continuity follows.

In case $\alpha-\mu=-1$, we just take any $\tau_{1}<1$ and the same argument applies.

Next, we are going to show that in the case $\alpha-\mu+1>0$ we can improve the global regularity of the solution, obtaining a Hölder estimate for the gradient in $\bar{\Omega}$. Here naturally the interesting case occurs when $\alpha-\mu<0$, since otherwise there is no singularity on the boundary, and the proof is much easier.

Theorem 4.2 Assume that $F$ satisfies $(S)$ and $(C)$, and that (3.1) holds, with $\alpha-\mu \in(-1,0)$. Then there exists $\beta \in(0,1)$ such that the solution $u$ of (1.1) is of class $C^{1, \beta}(\bar{\Omega})$.

In order to prove Theorem 4.2 we start with a preliminary result, which is an extension of Theorem 9.31 in [16] to viscosity solutions of fully nonlinear equations with singular right-hand sides.

Proposition 4.1 Assume F satisfies (HO)-(H3) and $u \in C^{0}\left(\bar{B}^{+}\right)$is a solution of

$$
F\left(D^{2} u, D u, u, x\right)=g(x) \quad \text { in } \quad B^{+}, \quad u=0 \quad \text { on } T,
$$

where $B^{+}:=B_{R_{0}} \cap \mathbb{R}_{+}^{N}$ and $T=B_{R_{0}} \cap \partial \mathbb{R}_{+}^{N}$, for some $R_{0} \leq 1$. Suppose that $u / x_{N}$ is bounded in $B^{+}$, and for some $a>0$

$$
\left|g\left(x^{\prime}, x_{N}\right)\right| \leq a x_{N}^{\alpha-\mu} \quad \text { in } \quad B^{+} .
$$

Then there are $R_{1} \in\left(0, R_{0}\right)$ and $\tau, C>0$ such that for all $0<R \leq R_{1}$

$$
\operatorname{Osc}_{B_{R}^{+}} \frac{u}{x_{N}} \leq C R^{\tau}
$$

Proof. Suppose first that $u \geq 0$. By (4.7) $g \in L_{\mathrm{loc}}^{\infty}\left(B^{+}\right)$, so $u \in W_{\mathrm{loc}}^{2, p}\left(B^{+}\right)$, for each $p<\infty$. As in [16] we start by proving that there exists $\delta>0$ such that

$$
\inf _{\substack{\left|x^{\prime}\right|<R \\ x_{N}=\delta R}} v \leq 2 \inf _{B_{R / 2, \delta}} v
$$

for any $R \leq R_{0}$, where

$$
v(x)=\frac{u(x)}{x_{N}} \quad \text { and } \quad B_{R, \delta}=\left\{x|| x^{\prime} \mid<R, \quad 0<x_{N}<\delta R\right\} .
$$

We may assume that the left hand side in (4.8) is positive, since otherwise the inequality is trivial, and we normalize so that $R=1$ and $\inf _{\substack{\left|x^{\prime}\right|<R \\ x_{N}=\delta R}} v=1$. 
Now we consider in $B_{1, \delta}$ the following barrier function

$$
w(x)=\left(1-\left|x^{\prime}\right|^{2}+\frac{x_{N}^{1-\nu}-\delta^{1-\nu}}{\delta^{(1-\nu) / 2}}\right) x_{N},
$$

where $\nu$ is some fixed number in the interval $(\mu-\alpha, 1)$. Then we compute

$$
\begin{gathered}
D w=\left(-2 x_{1} x_{N}, \ldots,-2 x_{N-1} x_{N}, 1-\left|x^{\prime}\right|^{2}+\delta^{(\nu-1) / 2}\left((2-\nu) x_{N}^{1-\nu}-\delta^{1-\nu}\right)\right), \\
D^{2} w(x)=\left[\begin{array}{ccccc}
-2 x_{N} & 0 & \ldots & 0 & -2 x_{1} \\
0 & -2 x_{N} & \ldots & 0 & -2 x_{2} \\
\vdots & & \ddots & \vdots \\
0 & \ldots & 0 & -2 x_{N} & -2 x_{N-1} \\
-2 x_{1} & \ldots & -2 x_{N-1} & b x_{N}^{-\nu}
\end{array}\right],
\end{gathered}
$$

with $b=(2-\nu)(1-\nu) /\left(\delta^{(1-\nu) / 2}\right)$. Note that $b$ is large when $\delta$ is small.

It is convenient to write $D^{2} w(x)=A+B$, where

$$
A:=\operatorname{diag}\left(-2 x_{N}, \ldots,-2 x_{N}, b(2-\nu)(1-\nu) x_{N}^{-\nu}\right)
$$

and the matrix $B$ has only two nontrivial eigenvalues, which are $\pm 2\left|x^{\prime}\right|$. To see this last point, take $\chi=(\tilde{x}, 0)$ with $\tilde{x} \perp x^{\prime}, \bar{x}_{ \pm}=\left(x^{\prime}, \pm\left|x^{\prime}\right|\right)$, and check that

$$
B(\chi)=0 \quad \text { and } \quad B \bar{x}_{ \pm}= \pm 2\left|x^{\prime}\right| \bar{x}_{ \pm} .
$$

It is simple to check that $x_{N} \in(0, \delta)$ implies $|w|,|D w| \leq C$ in $B_{1, \delta}$. Then we estimate

$$
\begin{aligned}
\mathcal{M}_{\lambda, \Lambda}^{-}\left(D^{2} w\right)-\gamma|D w| & \geq \mathcal{M}_{\lambda, \Lambda}^{-}(A)+\mathcal{M}_{\lambda, \Lambda}^{-}(B)-\gamma|D w| \\
& \geq-\Lambda x_{N}(N-1)+2(\lambda-\Lambda)\left|x^{\prime}\right|+\lambda b x_{N}^{-\nu}-C \\
& \geq 2 c_{0} x_{N}^{-\nu}-C \geq c_{0} x_{N}^{-\nu}
\end{aligned}
$$

provided $\delta$ is sufficiently small. On the other hand

$$
\mathcal{M}_{\lambda, \Lambda}^{-}\left(D^{2} u\right)-\gamma|D u| \leq F[u]+\gamma|u| \leq a x_{N}^{\alpha-\mu}+C \leq c_{0} x_{N}^{-\nu}
$$

since $\alpha-\mu>-\nu$, again for sufficiently small $\delta$. Finally, we observe that $w \leq 0$ on the lateral and the lower boundaries of $B_{1, \delta}$, while $w / x_{N} \leq 1 \leq u / x_{N}$ on the upper boundary of $B_{1, \delta}$. Hence $w \leq u$ on $\partial B_{1, \delta}$, and we can apply the usual comparison principle to the inequality

$$
\mathcal{M}_{\lambda, \Lambda}^{-}\left(D^{2} w\right)-\gamma|D w| \geq \mathcal{M}_{\lambda, \Lambda}^{-}\left(D^{2} u\right)-\gamma|D u|
$$


which holds in the strong sense in $B_{1, \delta}$. More precisely, $w-u \in W_{\text {loc }}^{2, p}\left(B_{1, \delta}\right) \cap$ $C\left(\overline{B_{1, \delta}}\right)$ satisfies $\mathcal{M}_{\lambda, \Lambda}^{+}\left(D^{2}(w-u)\right)+\gamma|D(w-u)| \geq 0$ in $B_{1, \delta}$ and hence is a solution of a linear inequality to which for instance Theorem 9.1 in [16] applies. So $u \geq w$ in $B_{1, \delta}$. Therefore

$$
v \geq\left(1-\left|x^{\prime}\right|^{2}+\frac{x_{N}^{1-\nu}-\delta^{1-\nu}}{\delta^{(1-\nu) / 2}}\right) \geq \frac{3}{4}-\delta^{1-\nu / 2} \geq \frac{1}{2} \quad \text { in } \quad B_{\frac{1}{2}, \delta},
$$

where the last inequality holds by making $\delta$ smaller, if necessary. By removing the normalization we get (4.8).

Define now the set

$$
B_{R / 2, \delta}^{*}:=\left\{x|| x^{\prime} \mid<R, \delta R / 2<x_{N}<3 \delta R / 2\right\} .
$$

Then by the Harnack inequality, applied in $B_{R / 2, \delta}^{*}$, we obtain

$$
\sup _{B_{R / 2, \delta}^{*}} u \leq C\left(\inf _{B_{R / 2, \delta}^{*}} u+R\left\|a x_{N}^{-\nu}\right\|_{L^{N}\left(B_{R / 2, \delta}^{*}\right)}\right) .
$$

But $\left\|a x_{N}^{-\nu}\right\|_{L^{N}\left(B_{R / 2, \delta}^{*}\right)} \leq C_{1} R^{1-\nu}$ and

$$
\frac{2}{3 \delta} \frac{u}{R} \leq v \leq \frac{2}{\delta} \frac{u}{R} \quad \text { in } \quad B_{R / 2, \delta}^{*}
$$

so, using (4.8) and dividing (4.9) by $R$, we obtain

$$
\begin{aligned}
\sup _{B_{R / 2, \delta}^{*}} v \leq C\left(\inf _{B_{R / 2, \delta}^{*}} v+C_{1} R^{1-\nu}\right) & \leq C\left(\inf _{\substack{|x|<R \\
x_{n}=\delta R}} v+C_{1} R^{1-\nu}\right) \\
& \leq C\left(\inf _{B_{R / 2, \delta}} v+C_{1} R^{1-\nu}\right) .
\end{aligned}
$$

Now we set $M=\sup _{B_{2 R, \delta}} v$ and $m=\inf _{B_{2 R, \delta}} v$. We define the positive functions $v_{1}=M-v, v_{2}=v-m$ and the associated $u_{1}=M x_{N}-u$, $u_{2}=u-m x_{N}$. Applying the above argument to $v_{1}$ and $v_{2}$ we get

$$
\sup _{B_{R / 2, \delta}^{*}}(M-v) \leq C\left(M-m_{1}+C_{1} R^{1-\nu}\right)
$$

and

$$
\sup _{B_{R / 2, \delta}^{*}}(v-m) \leq C\left(M_{1}-m+C_{1} R^{1-\nu}\right),
$$

where $M_{1}=\sup _{B_{R / 2, \delta}} v_{1}$ and $m_{1}=\sup _{B_{R / 2, \delta}} v_{1}$. Here we should notice that $u_{1}$ and $u_{2}$ satisfy elliptic equations with modified right-hand sides, which however satisfy (4.7) with $a$ replaced by $a+1$, provided we restrict to a sufficiently small neighbourhood of the boundary. Also, $u_{1}$ satisfies an equation with the operator $F$ replaced by $G[u]:=-F[-u]$, which also satisfies (H1). 
Adding the last two inequalities we obtain

$$
M-m \leq C\left(M-m_{1}+M_{1}-m+C_{1} R^{1-\nu}\right),
$$

which implies

$$
\operatorname{Osc}_{B_{R / 2, \delta}} v \leq \sigma\left(\operatorname{osc}_{B_{2 R, \delta}} v+C R^{1-\nu}\right),
$$

with $0<\sigma=(C-1) / C<1$. From this and Lemma 8.23 in [16] we obtain $\tau$ and $C$ with the desired property.

Proposition 4.2 Under the hypothesis of Proposition 4.1, there are numbers $R_{1} \in\left(0, R_{0}\right)$ and $\beta \in(0,1)$ such that $u \in C^{1, \beta}\left(\bar{B}_{R_{1}}^{+}\right)$.

Proof. It is a direct consequence of Proposition 4.1 that the function $u$ is differentiable on $T_{1}=B_{R_{1}} \cap \partial \mathbb{R}_{+}^{N}$ and that $D u(\cdot, 0) \in C^{\tau}\left(T_{1}\right)$. We observe that we also have

$$
\lim _{\left(x^{\prime}, x_{N}\right) \rightarrow\left(\bar{x}^{\prime}, 0\right)} \frac{\partial u\left(x^{\prime}, 0\right)}{\partial x_{N}}-\frac{u\left(x^{\prime}, x_{N}\right)}{x_{N}}=0,
$$

uniformly in $\left(\bar{x}^{\prime}, 0\right) \in T_{2}=B_{R_{2}} \cap \partial \mathbb{R}_{+}^{N}$, for any fixed $R_{2} \in\left(0, R_{1}\right)$.

Now, let us consider $\rho_{0}>0$ so that $\left(x^{\prime}, 6 \rho_{0}\right) \in B_{R_{1}}^{+}$for all $\left(x^{\prime}, 0\right) \in T_{2}$ and define $\tau_{1}=\min \{1-\nu, \tau\}$. Then for any fixed $\left(\bar{x}^{\prime}, 0\right) \in T_{2}$ we define $x_{\rho}=\left(\bar{x}^{\prime}, 3 \rho\right)$, for $0<\rho<\rho_{0}$, and the scaled function

$$
w(y)=\frac{1}{\rho^{1+\tau_{1}}}\left\{u\left(\rho y+x_{\rho}\right)-d_{0} \rho\left(y_{N}+3\right)\right\}, \quad y \in B_{3},
$$

where $d_{0}=d_{0}\left(\bar{x}^{\prime}\right):=\frac{\partial u\left(\bar{x}^{\prime}, 0\right)}{\partial x_{N}}$ is uniformly bounded. By Proposition 4.1

$$
u(x)-x_{N} d_{0}\left(\bar{x}^{\prime}\right) \leq C x_{N}^{1+\tau},
$$

so $w$ is a bounded function in $B_{3}$ satisfying

$$
F\left(D^{2} w, \rho D w+\rho^{1-\tau_{1}} d, \rho^{2} w+\rho^{2-\tau_{1}} d y_{N}, \rho y+x_{\rho}\right)=g_{\rho}(y),
$$

where $g_{\rho}(y)=g\left(\rho y+x_{\rho}\right) \rho^{1-\tau_{1}}$. By our choice of $\tau_{1}$ we see that $\left|g_{\rho}(y)\right| \leq c$ for all $y \in B_{2}$, with $c$ independent of $\rho$. Then the interior elliptic estimates for this problem give a constant $\beta \in(0,1)$ such that $w \in C^{1, \beta}\left(B_{1}\right)$, that is, for some constant $C$ we have

$$
\left|D w\left(y_{1}\right)-D w\left(y_{2}\right)\right| \leq C\left|y_{1}-y_{2}\right|^{\beta}, \quad \text { for all } y_{1}, y_{2} \in B_{1} .
$$


Here, as in Theorem 4.1, $\beta$ and $C$ are independent of $\rho$ and $x^{\prime} \in T_{2}$. We decrease $\beta$, if necessary, so that $\beta \leq \tau_{1}$ and we see that, by the definition of $w$,

$$
\left|D u\left(x_{1}\right)-D u\left(x_{2}\right)\right| \leq C\left|x_{1}-x_{2}\right|^{\gamma}, \quad \text { for all } \quad x_{1}, x_{2} \in B\left(x_{\rho}, \rho\right),
$$

where the estimate is uniform in $\rho \in\left(0, \rho_{0}\right)$ and $\bar{x}^{\prime} \in T_{2}$. Hence we can prove, following the same steps as in the proof of Theorem 4.1, that for any given $\bar{x}^{\prime}$ the one dimensional function $z(s)=D u\left(\bar{x}^{\prime}, s\right)$ satisfies

$$
\left|z\left(s_{1}\right)-z\left(s_{2}\right)\right| \leq C\left|s_{1}-s_{2}\right|^{\gamma}, \quad \text { for all } \quad s_{1}, s_{2} \in\left(0,4 \rho_{0}\right)
$$

where the constant $C$ can be chosen independent of $\bar{x}^{\prime} \in T_{2}$.

To extend this to $s_{1}=0$ we need to prove that the gradient of $u$ is continuous in $\bar{B}_{R_{2}}^{+}$. Observe that

$$
\begin{aligned}
\left|D u\left(x^{\prime}, x_{N}\right)-\frac{u\left(x^{\prime}, x_{N}\right)}{x_{N}}\right| & =\left|D u\left(x^{\prime}, x_{N}\right)-D\left(x^{\prime}, \xi\right)\right| \\
& \leq C\left|x_{N}-\xi\right|^{\gamma} \leq C\left|x_{N}\right|^{\gamma}
\end{aligned}
$$

where we used the mean value theorem to find $\xi \in\left(0, x_{N}\right)$ and then (4.12), recalling that this inequality holds uniformly in $x^{\prime} \in T_{2}$. Hence if $\left(\bar{x}^{\prime}, 0\right) \in T_{2}$, then by

$$
D u\left(x^{\prime}, x_{N}\right)-D\left(\bar{x}^{\prime}, 0\right)=D u\left(x^{\prime}, x_{N}\right)-\frac{u\left(x^{\prime}, x_{N}\right)}{x_{N}}+\frac{u\left(x^{\prime}, x_{N}\right)}{x_{N}}-D\left(\bar{x}^{\prime}, 0\right),
$$

we get the continuity, thanks to (4.10) and (4.13).

Therefore we can extend (4.12) to all $s \in\left[0,4 \rho_{0}\right]$, exactly as in Theorem 4.1. Then we can finally prove the global Hölder continuity in $\bar{B}_{R_{2}}^{+}$as follows. Given $x=\left(x^{\prime}, x_{N}\right), y=\left(y^{\prime}, y_{N}\right) \in \bar{B}_{R_{2}}^{+}$, we consider two cases:

i) If we have $|x-y|<x_{N} / 3$ or $|x-y|<y_{N} / 3$ we just apply (4.11) in a ball containing both $x$ and $y$.

ii) Otherwise we have, by using (4.12),

$$
\begin{aligned}
& \frac{\left|D u(x)-D u\left(x^{\prime}, 0\right)\right|}{|x-y|^{\gamma}} \leq 3^{\gamma} \frac{\left|D u(x)-D u\left(x^{\prime}, 0\right)\right|}{x_{N}^{\gamma}} \leq 3^{\gamma} C, \\
& \frac{\left|D u(y)-D u\left(y^{\prime}, 0\right)\right|}{|x-y|^{\gamma}} \leq 3^{\gamma} \frac{\left|D u(y)-D u\left(y^{\prime}, 0\right)\right|}{x_{N}^{\gamma}} \leq 3^{\gamma} C,
\end{aligned}
$$

and

$$
\frac{\mid D u\left(x^{\prime}, 0\right)-D u\left(y^{\prime}, 0\right)}{|x-y|^{\gamma}} \leq \frac{\mid D u\left(x^{\prime}, 0\right)-D u\left(y^{\prime}, 0\right)}{\left|x^{\prime}-y^{\prime}\right|^{\gamma}} C,
$$


where we used $|x-y| \geq\left|x^{\prime}-y^{\prime}\right|$ and the Hölder continuity of $D u$ on $T_{2}$, as mentioned at the beginning of the proof. Here may need to decrease $\gamma$, if necessary to have $\gamma \leq \tau$.

Acknowledgements: We thank the anonymous referee for many useful remarks.

P.F. was partially supported by Fondecyt Grant \# 1070314, FONDAP and BASAL-CMM projects and MathAmsud 08MATH01.

A. Q. was partially supported by Fondecyt Grant \# 1070264 and USM Grant \# 12.09.17. and Programa Basal, CMM. U. de Chile.

\section{References}

[1] S. Armstrong, Principal eigenvalues and an anti-maximum principle for nonlinear elliptic equations. J. Differential Equations 246 (2009) 2958-2987.

[2] M. Bardi, F. Da Lio, On the strong maximum principle for fully nonlinear degenerate elliptic equations, Arch. Math. (Basel) 73 (1999) 276-285.

[3] X. Cabre, Elliptic PDEs in probability and geometry, Discr. Cont. Dyn. Syst. A, 20(3) (2008), 425-457.

[4] X. Cabré, L.A. Caffarelli, Fully Nonlinear Elliptic Equations, American Mathematical Society, Colloquium Publications, 43 (1995).

[5] L.A. Caffarelli, M.G. Crandall, M.Kocan, A. Świech, On viscosity solutions of fully nonlinear equations with measurable ingredients, Comm. Pure Al. Math 49 (1996), 365-397.

[6] F. Cirstea, M. Ghergu and V. Radulescu, Combined effects of asymptotically linear and singular nonlinearities in bifurcation problems of LaneEmden type, J. Math. Pures Appl. 84 (2005), 493-508.

[7] M.M. Coclite and G. Palmieri, On a singular nonlinear Dirichlet problem, Commun. Partial Differential Equations 14 (1989), 1315-1327.

[8] M. Crandall, H. Ishii and P.L. Lions. User's guide to viscosity solutions of second order partial differential equations. Bulletin of the AMS, 27(1) (1992), 1-67.

[9] M.G. Crandall, M. Kocan, P.L. Lions, A. Świech, Existence results for boundary problems for uniformly elliptic and parabolic fully nonlinear equations, Elec. J. Diff. Eq. 24 (1999), 1-20. 
[10] M.G. Crandall, P.H. Rabinowitz and L. Tartar, On a Dirichlet problem with singular nonlinearity, Commun. Partial Differential Equations 2 (1977), 193-222.

[11] J. Davila and M. Montenegro, Positive versus free boundary solutions to a singular equation, J. Anal. Math. 90 (2003), 303-335.

[12] M. del Pino, A global estimate for the gradient in a singular elliptic boundary value problem, Proc. Roy. Soc. Edinburgh Sect. A 122 (1992), 341-352.

[13] J.I. Diaz, J.M. Morel and L. Oswald, An elliptic equation with singular nonlinearity, Commun. Partial Differential Equations 12 (1987), 1333-1344.

[14] M. Esteban, P. Felmer, A. Quaas. Super-linear elliptic equation for fully nonlinear operators without growth restrictions for the data. Proc. Roy. Soc. Edinburgh Sect. A, to appear

[15] W.H. Fleming. H. Mete Soner, Controlled Markov Processes and Viscosity Solutions, 2nd edition, vol. 25 of Stochastic Modelling and Probability, Springer-Verlag, 2005.

[16] D. Gilbarg, N. Trudinger, Elliptic partial differential equations of second order. Reprint of the 1998 edition. Classics in Mathematics. SpringerVerlag, Berlin, (2001).

[17] S.N. Gomes, On a singular nonlinear elliptic problem, SIAM J. Math. Anal. 17 (1986), 1359-1369.

[18] C. Gui and F. Hua Lin, Regularity of an elliptic problem with a singular nonlinearity, Proc. Roy. Soc. Edinburgh Sect. A 123 (1993), 1021-1029.

[19] J. Hernandez, F.J. Mancebo, Singular elliptic and parabolic equations, in Handbook of Differential Equations, Stationary PDE, vol. 3, ChipotQuittner Eds, Elsevier, (2006), 317-400.

[20] J. Hernandez, F.J. Mancebo and J.M. Vega, On the linearization of some singular nonlinear elliptic problems and applications, Ann. Inst. H. Poincar Anal. Non Linaire 19 (6) (2002), 777-813.

[21] J. Hernandez, F.J. Mancebo and J.M. Vega, Positive solutions for singular nonlinear elliptic equations, Proc. Roy. Soc. Edinburgh Sect. A 137(1) (2007), 41-62.

[22] H. Ishii, P.-L. Lions, Viscosity solutions of fully nonlinear second-order elliptic partial differential equations, J. Diff. Eq. 83 (1990), 26-78.

[23] N. V. Krylov, Nonlinear elliptic and parabolic equations of the second order. Mathematics and its Applications (Soviet Series), 7. D. Reidel Publishing Co., Dordrecht, (1987). 
[24] N.V. Krylov, Fully nonlinear second order elliptic equations : recent development, Ann. Sc. Norm. Pisa 25(3-4) (1997), 569-595.

[25] A. Lazer, P. McKenna, On a singular nonlinear elliptic boundary value problem, Proc. Amer. Math. Soc. 111 (1991), 721-730.

[26] P.L. Lions, Bifurcation and optimal stochastic control, Nonl. Anal. Th. and Appl. 7(2) (1983), 177-207.

[27] N. Nadirashvili, Nonuniqueness in the martingale problem and the Dirichlet problem for uniformly elliptic operators. Ann. Scuola Norm. Sup. Pisa Cl. Sci. 24(3) (1997), 537-549.

[28] A. Quaas, B. Sirakov, Existence results for nonproper elliptic equation involving the Puccis Operator. Comm. Partial Differential Equations, 31(7) (2006), 987-1003.

[29] A. Quaas, B. Sirakov, Principal eigenvalues and the Dirichlet poblem for fully nonlinear elliptic operators. Adv. Math. 218 (2008), 105-135.

[30] P. H. Rabinowitz. Théorie du degré topologique et applications à des problèmes aux limites non linéaires, Lecture Notes, Lab. Analyse Numérique, Université PARIS VI, (1975).

[31] J. Shi and M. Yao, On a singular nonlinear semilinear elliptic problem, Proc. Royal Soc. Edinburgh Sect. A 138 (1998), 1389-1401.

[32] B. Sirakov, Solvability of Uniformly Elliptic Fully Nonlinear PDE, Arch. Rat. Mech. Anal., to appear, DOI : 10.1007/s00205-009-0218-9.

[33] H. M. Soner, Stochastic representations for nonlinear parabolic PDEs, survey article, people.sabanciuniv.edu/msoner/publications (2007).

[34] C.A. Stuart, Existence and approximation of solutions of nonlinear elliptic equations, Math. Z. 147 (1976), 53-63.

[35] A. Świech, $W^{1, p}$-estimates for solutions of fully nonlinear uniformly elliptic equations, Adv. Diff. Eq. 2(6) (1997), 1005-1027.

[36] N. Winter, $W^{2, p}$ and $W^{1, p}$-estimates at the boundary for solutions of fully nonlinear, uniformly elliptic equations, Z. Anal. Adwend. (J. Anal. Appl.) 28(2) (2009), 129-164.

[37] S. Yijing, W. Shaoping and L. Yiming, Combined effects of singular and superlinear nonlinearities in some singular boundary value problems, J. Differential Equations 176 (2001), 511-531.

[38] Z. Zhang and J. Yu, On a singular nonlinear Dirichlet problem with a convection term, SIAM J. Math. Anal. 32 (2000), 916-927. 
Patricio Felmer, Departamento de Ingeniería Matemática and Centro de Modelamiento Matemático, UMR2071 CNRS-UChile Universidad de Chile, Casilla 170 Correo 3, Santiago, Chile. (pfelmer@dim.uchile.cl)

Alexander Quaas, Departamento de Matemática, Universidad Santa María Casilla: V-110, Avda. España 1680, Valparaíso, Chile. (alexander.quaas@usm.cl)

Boyan Sirakov, UFR SEGMI, Université de Paris 10, 92001 Nanterre Cedex, France, and CAMS, EHESS, 54 bd. Raspail, 75006 Paris, France (sirakov@ehess.fr) 